\title{
Clinical reasoning and COVID 19 pandemic: current influencing factors Let us take a step back!
}

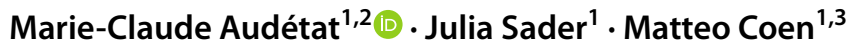

Received: 30 June 2020 / Accepted: 24 September 2020 / Published online: 12 October 2020

(c) Società Italiana di Medicina Interna (SIMI) 2020

\author{
Caminante, no hay camino, se \\ hace camino al andar. \\ Traveller, there is no path, paths \\ are made by walking. \\ Antonio Machado (free \\ translation)
}

Clinical reasoning may be defined as "the sum of the thinking and decision-making processes associated with clinical practice (...) it enables practitioners to take "wise" action, making the best-judged action in a specific context" [1]. Although perfectible, these reasoning processes are relatively well established in clinical settings and teams of health care professionals. In the last few weeks, COVID-19 has become the leading cause of hospitalization, morbidity and mortality in most of the world [2].

The fight against this pandemic has shaken up clinical practices, also regarding quality improvement and patient safety. Uncertainties related to the virus itself, influx of patients deteriorating very quickly, the need to develop new ways of working, changes in teams and work schedules, but also stress, fatigue and fear for oneself and for loved ones, are all elements of the context which contribute to influencing clinical reasoning. From then on, in such an uncertain, rapidly changing and potentially anxiety-provoking context, would it not be worthwhile to think about how our clinical reasoning may be impacted? Authors sometimes use the term problem space to reflect these multiple contexts and

Marie-Claude Audétat

Marie-Claude.Audetat@unige.ch

1 (UDREM) Unit of Development and Research in Medical Education, Faculty of Medicine, University of Geneva, Geneva, Switzerland

2 (UIGP) Primary Care Unit, Faculty of Medicine, University of Geneva, Geneva, Switzerland

3 Division of General Internal Medicine, Department of Medicine, Geneva University Hospitals, Geneva, Switzerland their specificities. "Problem spaces comprise the immediate clinical problem and task environment of clinical decision making embedded in the interests and frames of references of practitioners and patient" [1]. These problem spaces are part of the broader clinical reasoning context that encompasses the local, organisational, sociocultural and global factors that influence clinical reasoning. Here is a clinical example to illustrate how issues related to these spaces can affect clinical reasoning (Fig. 1).

A 70-year-old man consulted for profound fatigue. The patient's medical history was significant for aortic valve replacement in the past year. The patient was ill looking and highly febrile; physical examination revealed a systolic murmur over the aortic valve area and crackles over the lower lobes of lungs. Laboratory tests showed leucocytosis, and elevated C-reactive protein and venous lactate. ECG and chest X-ray were normal. Two nasal swabs for severe acute respiratory syndrome coronavirus 2 (SARS-CoV-2) turned out negative; blood cultures were drawn. Despite the negative swabs, and without evidence of COVID-19 pneumonia, a SARS-CoV-2 infection was considered as the most likely. The patient was transferred to a ward for stable patients with COVID-19, without antibiotic treatment. A few hours later the patient became hemodynamically unstable. Physical examination was unchanged, besides an erythematous lesion of the hallux (that was later found to be an Osler's nodule). Blood cultures turned positive for Staphylococcus aureus. Clinical and biological features were suggestive for infectious endocarditis. Transoesophageal echocardiography showed an abscess in the aortic annulus. Broad-spectrum antibiotics were administered, and emergent cardiac surgery planned.

How come this patient (presenting all the signs and symptoms related to the well-known and classic illness script of endocarditis) was diagnosed COVID-19 for several hours and by two successive teams of senior doctors? Looking at this case after-the-fact gives a different perspective, for example: the high rate of false negatives of the swab tests. 


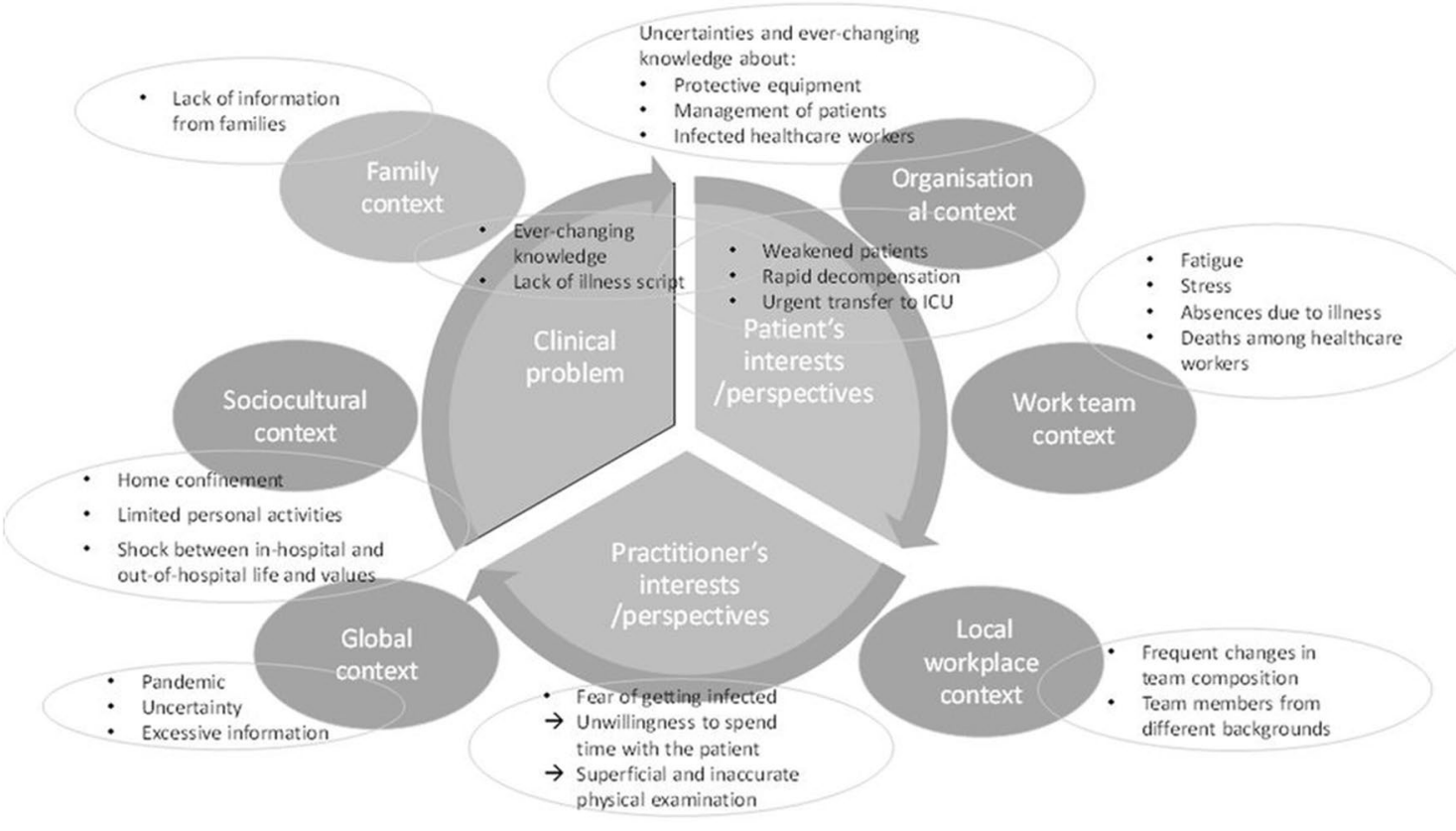

Fig. 1 Potential challenges and factors influencing clinical reasoning (adapted from Higgs [1])

Clinicians should have a global representation of the clinical case and should integrate into their differential diagnosis the notion de probabilities and prioritization $[3,4]$.

Dynamic settings potentially influence clinical reasoning and decision making [1]. This is particularly the case nowadays with COVID-19: clinical problems are ill-defined and made ambiguous by the presence of incomplete ongoing information and development of new knowledge. Moreover, multiple healthcare professionals are acting in teams who normally do not work together as they integrate volunteers (medical students, military personnel) to respond to this pandemic. As a result, the environment is uncertain, and decision-making may change from day to day. Keeping in mind that all of this is part of a global context where there remains a gap between the health care professionals in the front line working in the midst of a pandemic and the rest of the population self-isolating, often working from home, isolated from their families, having to adapt to this new challenging reality. These changing contexts and their interactions are more likely to lead to errors in clinical reasoning. Clinicians are accustomed to the following metaphor that reminds them of the importance of incidence and prevalence when making diagnosis: "If you hear the sound of hoof beats, think horses, not zebras" [5]. But in this case, the usual cues are confused. A troop of zebras has arrived in the city... you hear hoofbeats everywhere... Horses? Zebras? Interpreting clinical signs and symptoms is a challenge, as is prioritizing them for diagnosis and management. In this example, clinicians tended to interpret the information gained during a consultation to fit their preconceived diagnosis, acting the so-called premature closure. Premature closure is a very common difficulty amongst the various clinical reasoning problems which may be identified when clinicians seek only the data which confirm their single hypothesis, failing to notice new cues and/or failing to explore cues or information which could lead to other diagnostic hypotheses [6]. Cognitive biases, such as confirmation bias, or anchoring bias might be the cause of this difficulty.

To date, there is no defined COVID-19 illness script; for example, clinical characteristics of COVID-19 patients are atypical: contrary to common clinical tenets, COVID-19 patients may be extremely hypoxemic and yet present no dyspnea. As we are constructing the COVID's illness script, any clinical encounter, each discussion with colleagues potentially enriches this illness script. This requires reflective practice and excellent clinical and reasoning skills. In other words, taking the time to think about the multitude of factors including the human ones. In addition, it is key to keep in mind the importance of the rigorous hypotheticodeductive process which can sometimes be put aside during a health crisis.

A step back should then be taken, accepting that one cannot be sure, developing one's ability to tolerate but also to share one's uncertainty with others. As Moulton suggested, one might consider that expert judgement is about the ability to "slow down when appropriate and take the time to ensure that the muddy problems of practice will be correctly named and framed" [7]. Namely, it may be a matter of continuing to think and ask oneself: what if it was something else? Or assigning a colleague in the team to be the "devil's 
advocate" to reassess the clinical reasoning process and avoid making decisions too quickly and unilaterally.

In conclusion, while clinicians from all around the world are building the illness script of COVID-19, it is therefore key to keep in mind that clinical reasoning is a journey, an aspiration and a commitment to achieve the best practice that one can provide [1].

\section{Compliance with ethical standards}

Conflict of interest The authors declare that they have no conflict of interest.

Statement of human and animal rights This article does not contain any studies with human participants or animals performed by any of the authors.

Informed consent None.
Reasoning in the Health Professions, 3rd edn. Butterworth-Heineman Ldt, Oxford

2. Verity R, Okell LC, Dorigatti I, Winskill P, Whittaker C, Imai N et al (2020) Estimates of the severity of coronavirus disease 2019: a model-based analysis. Lancet Infectious Dis 20(6):669-677

3. Nendaz M, Perrier A (2004) Théorème de Bayes et rapports de vraisemblance [Bayes theorem and likelihood ratios]. Rev Mal Respir 21:394-397

4. Westbury C (2010) Bayes' rule for clinicians: an introduction. Front Psychol 1(192):1-7

5. Colgan R (2009) Advice to the young physician : on the art of medicine. Springer, New York

6. Audétat M-C, Laurin S, Dory V, Charlin B, Nendaz M (2018) Diagnosis and management of clinical reasoning difficulties: AMEE GUIDE 117. Association for Medical Education in Europe (AMEE), Dundee

7. Moulton C, Regher G, Mylopoulos M, MacRae H (2007) Slowing down when you should: a new model of expert judgment. Acad Med 82(10):S109-S116

Publisher's Note Springer Nature remains neutral with regard to jurisdictional claims in published maps and institutional affiliations.

\section{References}

1. Higgs J, Jones M (2008) Chapter 1: Clinical decision making and multiple problem spaces. In: Higgs J, Jones M (eds) Clinical 